\title{
Desenvolvimento e validação de vídeo educativo para autocateterismo vesical intermitente limpo
}

\author{
Development and validation of an educational video for clean intermittent \\ bladder catheterization
}

\author{
Fabiana Faleiros ${ }^{1}$, Cibele Dias Cucick ${ }^{1}$, Elias Tristão da Silva Neto을 Soraia Assad Nasbine Rabeh', \\ Naira Beatriz Favoretto², Christoph Käppler ${ }^{2}$
}

\begin{abstract}
RESUMO
Trata-se do desenvolvimento e validaçáo de um vídeo educativo sobre autocateterismo vesical intermitente limpo. Estudo metodológico, realizado em três etapas: pré-produção (elaboração e validação do roteiro e storyboard), produção do vídeo, e pós-produção (validação do vídeo por juízes experts em reabilitação e/ou saúde). O roteiro para produção do vídeo foi desenvolvido e validado por 18 juízes, com $91,1 \%$ de concordância. O vídeo, com 10 minutos e cinco segundos, também foi validado e atingiu 97,4\% de concordância entre os 17 juízes participantes nos quesitos funcionalidade, usabilidade, eficiência, técnica audiovisual, ambiente e procedimentos. A versão final do vídeo desenvolvido e validado pode ser visualizada no Portal do Núcleo de Pesquisa NEUROREHAB, www.demaisinformacao.com.br. Essa ferramenta pode contribuir para a capacitação de pessoas com bexiga neurogênica a realizar o autocateterismo urinário, bem como profissionais de saúde e estudantes de enfermagem, e apoiar metodologicamente o desenvolvimento de outros vídeos educativos na área da saúde.
\end{abstract}

Descritores: Bexiga Urinaria Neurogênica; Cateterismo Urinário; Reabilitação; Filmes e Vídeos Educativos; Enfermagem.

\section{ABSTRACT}

The purpose of this study was to develop and validate an educational video on clean intermittent self-catheterization. It was a methodological study, carried out in three stages: pre-production (elaboration and validation of the script and storyboard), video production, and post-production (validation of the video by expert judges working in the field of rehabilitation and/or health). The script for video production was developed and validated by 18 judges, with $91.1 \%$ agreement. The video, with a duration time of 10 minutes and five seconds, was also validated and reached $97.4 \%$ of agreement among the 17 participating judges regarding functionality, usability, efficiency, audiovisual technique, environment and procedures. The final version of the video developed and validated can be viewed at the NEUROREHAB Research Center Portal, https://demaisinformacao.com.br/ autocatetrismourinario/. This tool can contribute to the training of people with neurogenic bladder, as well as health professionals and nursing students, to perform self-catheterization, and methodologically support the development of other educational videos in the health area.

Descriptors: Neurogenic Bladder; Self-catheterization; Rehabilitation; Instructional Films and Videos; Nursing.

\footnotetext{
${ }^{1}$ Universidade de São Paulo _ Ribeirão Preto (SP), Brasil. E-mails: fabifaleiros@eerp.usp.br, cicucick@gmail.com, elias.tristao.neto@usp.br, soraia@eerp.usp.br ${ }^{2}$ Universidade Tecnológica de Dortmund — Dortmund (Renânia do Norte-Vestfália), Alemanha. E-mails: nairafavoretto@gmail.com, christoph.kaeppler@, $\underline{\text { tu-dortmund.de }}$
}

Agradecimentos: à Fundação de Amparo à Pesquisa do Estado de São Paulo (FAPESP) e à Empresa Coloplast.

Como citar este artigo: Faleiros F, Cucick CD, Silva Neto ET, Rabeh SAN, Favoretto NB, Käppler C. Rev. Eletr. Enferm. Desenvolvimento e validação de vídeo educativo para autocateterismo vesical intermitente limpo. [Internet]. 2019 [acesso em: ]; 21:53973. Disponível em: https://doi.org/10.5216/ree.v21.53973. 


\section{INTRODUÇÃO}

A bexiga neurogênica $(\mathrm{BN})$ tem como complicações principais a incontinência urinária, a infecção do trato urinário, o refluxo vesicoureteral e a hidronefrose, as quais, quando não prevenidas e tratadas, podem levar à deterioraçáo renal e até mesmo ao óbito ${ }^{(1,2)}$. Quando confirmada a condição de $\mathrm{BN}$, o cateterismo vesical ou urinário intermitente limpo deve ser realizado de quatro a seis vezes ao dia, durante toda a vida, visando a proteção do trato urinário superior ${ }^{(3,4)}$.

Nesse sentido, o autocateterismo vesical intermitente (ACVI) é essencial para a preservação da intimidade, privacidade, sexualidade, aquisição de autonomia, inclusão e participação na sociedade. Contudo, a capacitação para o cateterismo intermitente $(\mathrm{CI})$, em especial para o autocateterismo, é um desafio enfrentado por essas pessoas, suas famílias e também por profissionais de saúde ${ }^{(5,6)}$.

Diante desses desafios se torna indispensável a realização de açóes que promovam a capacitação, o apoio e a motivação das pessoas com $\mathrm{BN}$ e seus familiares para a realização do $\mathrm{ACVI}^{(5)}$. Essas açóes devem promover o incentivo ao autocateterismo vesical intermitente; a disponibilidade de cuidadores ou enfermeiros para realização da técnica regularmente durante o dia no caso de pessoas dependentes; a promoção e melhoria de políticas públicas para a acessibilidade à instalaçôes sanitárias para realização do CI; a escolha correta do cateter, com melhor qualidade e adaptado às necessidades e experiências de cada indivíduo; a prevenção de eventos adversos durante a realização do CI; e o desenvolvimento de estratégias que auxiliem o processo de ensino-aprendizagem do autocateterismo vesical intermitente ${ }^{(3-6)}$.

Nessa direção, a enfermagem tem avançado na elaboração de ferramentas educativas e buscado no ambiente virtual (AV) um meio de socialização do conhecimento, colaboração para a melhoria da promoção do saber empírico estruturado em pesquisas científicas e criação de uma ponte mais abrangente de interação enfermeiro-paciente ${ }^{(7-10)}$. $\mathrm{O} \mathrm{AV}$ fortalece a atuação autônoma do indivíduo na produção do seu conhecimento, ao mesmo tempo em que proporciona apoio e uma aprendizagem coletiva, com a disponibilidade de não se deslocar de seu ambiente familiar, superando barreiras geográficas, físicas e ambientais ${ }^{(7-10)}$.

Considerando a realidade cibernética, o vídeo educativo (VE) é um instrumento de ensino-aprendizagem que aproxima o ambiente educacional das relaçóes cotidianas, das linguagens e dos códigos utilizados pela populaçãao ${ }^{(11)}$. Apesar disso, são escassos os vídeos para o aprendizado da autocateterizaçáo no contexto brasileiro, disponíveis na internet, que levem em consideração os cateteres urinários disponíveis e a técnica utilizada no Brasil ${ }^{(12)}$.

Diante disso, o objetivo deste estudo foi desenvolver e validar um vídeo educativo para o aprendizado da técnica autocateterismo vesical intermitente limpo, feminino e masculino, com o uso da tecnologia $3 \mathrm{D}$ e avatares.

\section{MÉTODOS}

Trata-se de um estudo metodológico que engloba o processo de desenvolvimento e validação de um VE para aprendizagem do autocateterismo vesical intermitente com a técnica limpa, contemplando a elaboração e validação do roteiro do VE, a produção do Storyboard, a produção do vídeo e a validação do $\mathrm{VE}^{(9,10)}$.

Esta pesquisa foi autorizada e aprovada pelo Comitê de Ética da Escola de Enfermagem de Ribeirão Preto, da Universidade de São Paulo sob o número CAAE no 35095214.8.0000.5393. Todos os participantes aceitaram o Termo de Consentimento Livre e Esclarecido (TCLE) para a participação na pesquisa científica.

Inicialmente foi feito um levantamento da produção dos VEs voltados para o ensino do autocateterismo vesical intermitente disponíveis no site de compartilhamentos de vídeos gratuito, Youtube ${ }^{\circledR}$, o mais divulgado e utilizado pela população $^{(13)}$. O descritor utilizado foi "autocateterismo", somente em português, no Brasil, uma vez que o estudo buscou o conteúdo voltado ao público brasileiro. Os critérios de inclusão para seleção dos vídeos avaliados foram: vídeos sobre o ACVI, com a técnica limpa e em língua portuguesa.

Os vídeos foram analisados por três juízes com experiência $^{(13)}$ na capacitação para a realização da técnica, utilizando um questionário adaptado de avaliação de vídeos validado por Ferreira ${ }^{(14,15)}$.

Para a avaliação dos vídeos do Youtube $^{\circledR}$ e para o desenvolvimento do vídeo foi considerada a técnica de autocateterização intermitente limpa, foi baseada em diretrizes da sociedade internacional (Sociedade Brasileira de Urologia, Associaçáo Européia de Urologia e American Urological Association), adaptada à realidade brasileira e recomendada

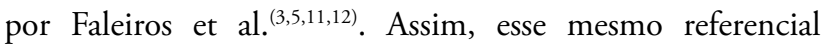
foi utilizado para a criação do vídeo, considerando que os materiais analisados a partir do Youtube ${ }^{\circledR}$ não atenderam aos critérios de qualidade, como descritos na seção Resultados.

Em seguida, elaborou-se o roteiro do VE e a produção do Storyboard para permitir a visualização global prévia do vídeo, o planejamento das cenas da animação e experimentação de diversas técnicas ${ }^{(16,17)}$. Além de possibilitar a gravação dos diálogos e a reformulaçáo de cenas. Durante sua concepção utilizou-se o programa de edição de imagens Photoshop ${ }^{\circledR}$.

Para a seleção dos juízes deste estudo foi considerado o sistema de classificação de experts a partir dos critérios adotados por Fehring e pesquisadores ${ }^{(18-20)}$, considerando experts apenas os participantes que alcançassem uma pontuação mínima de cinco pontos, seguindo a lógica de pontuação: Título de doutor $=4$ pontos, Título de mestre $=3$ pontos, publicação em periódico indexado sobre a temática de interesse do estudo $=2$ pontos, Especialização na temática de interesse do estudo $=2$ pontos, Prática clínica na área de interesse de no mínimo cinco anos na área de reabilitação $=2$ pontos, 
Participação em evento científico nos últimos dois anos sobre a temática de interesse do estudo $=1$ ponto. Tanto para a validação do roteiro quanto para validação do $\mathrm{VE}$, foram convidados os mesmos 34 juízes selecionados pela amostragem intencional e bola de neve. No entanto, 18 juízes aceitaram participar da validaçáo do roteiro e 17 aceitaram validar o VE.

Para a análise do roteiro educativo junto aos experts, foi utilizado um questionário validado em estudos anteriores e adaptado à temática do autocateterismo vesical intermitente ${ }^{(8,16,17)}$. Assim, realizou-se a validação de conteúdo do roteiro do VE por 18 experts, os quais foram contatados por e-mail. A escolha destes se deu por meio da amostragem intencional, a qual permite a escolha voluntária de especialistas na temática do estudo para atuarem como juízes da validaçáo. Optou-se pela seleção de profissionais de redes de reabilitação e pesquisadores de diferentes universidades tendo como exigência a experiência prática ou acadêmica de no mínimo cinco anos na temática da pesquisa.

O instrumento de validaçáo do roteiro do vídeo educativo foi dividido nos seguintes quesitos: objetivos (propósitos do VE), conteúdo (apresentação, forma e estrutura do VE), relevância (características de imagens e cenas propostas no roteiro do $\mathrm{VE}$ ), ambiente (avaliação do cenário do $\mathrm{VE}$ ), linguagem verbal (avaliação da linguagem empregada) e inclusão de tópicos (nesse quesito os juízes podiam sugerir a inclusôes no roteiro), composto por 19 questóes, sendo 16 questóes de múltipla escolha com um campo aberto para sugestóes e três questôes abertas. Foi utilizada a escala Likert ${ }^{(14,15)}$ de cinco pontos, onde os juízes deveriam escolher a opção que corresponde sua opinião. Os questionários de validação foram elaborados e respondidos virtualmente, utilizando a plataforma virtual Survey Monkey ${ }^{\circledR}$.

Para a produção do VE, foi contratada uma empresa especializada na produção de vídeos com tecnologia $3 \mathrm{D}$ e mídias educativas, composta por um designer gráfico, um motion designer, um modelador $3 \mathrm{D}$, dois animadores $3 \mathrm{D}$, dois locutores, um produtor de vídeo e um editor de vídeo.

Em seguida foram convidados novamente por $e$-mail, juízes previamente selecionados para validação do VE, levando em consideraçáo o mesmo critério de seleçáo utilizado na seleção dos juízes para validação do roteiro do VE. O e-mail enviado continha o link do questionário de validação do VE e do vídeo disponível para download, arquivos com o roteiro validado do VE e o TCLE, e mensagem de estimativa de preenchimento em 30 a 50 minutos. O questionário de validação do VE contemplou as seguintes sessóes: caracterização dos juízes, funcionalidade, usabilidade, eficiência, técnica audiovisual, ambiente e procedimento.

Para a análise estatística descritiva foi realizado o cálculo de frequência e porcentagem, o cálculo da média e desvio-padrão $( \pm)$. As versóes finais do roteiro e do VE só foram consideradas validadas quando $70 \%$ dos juízes atribuíram o conceito "concordo totalmente" e/ou "concordo" em cada item avaliado, conforme critério adotado em estudos anteriores $^{(7,8,21)}$. Os itens avaliados pelos juízes que não atingiram $70 \%$ de concordância das respostas nos conceitos "concordo totalmente" e/ou "concordo" foram analisados e reformulados ${ }^{(7,8,21)}$.

\section{RESULTADOS}

Os resultados serão descritos respeitando a ordem cronológica utilizada para o desenvolvimento e validação do VE, conforme relatado no método.

\section{Desenvolvimento}

O desenvolvimento de um novo vídeo foi necessário ao identificar lacuna na revisão de outras mídias disponíveis no YouTube $^{\circledR}$. Na busca, surgiram 174 vídeos, que após aplicação dos critérios de inclusão culminaram em apenas seis.

Os vídeos avaliados não apresentaram todas as informaçôes necessárias quanto à fisiopatologia da $\mathrm{BN}$ e/ou demonstraram técnica incorreta, incompleta ou desatualizada, por vezes devido à falta de materiais que não são disponibilizados pelos serviços de reabilitação no Brasil, outras incompatíveis com a técnica utilizada no contexto brasileiro. Considerando a lacuna e o risco de não informar corretamente a população alvo, comprovou-se a necessidade de produção de materiais educativos para aprendizagem do ACVI, baseados em evidências científicas e sem fins publicitários.

A produção do VE teve duração de três meses e resultou em um material com duração de 10 minutos e cinco segundos, abrangendo os seguintes tópicos: apresentação dos personagens; anatomia e fisiologia do sistema urinário; fisiopatologia da $\mathrm{BN}$; complicaçôes da $\mathrm{BN}$; descrição dos materiais e da técnica do autocateterismo vesical intermitente limpo masculino e feminino.

A narração foi gravada em um studio especializado em locução, sendo posteriormente transferido para o aplicativo Audacity ${ }^{\circledR}$ onde a narração foi inserida no vídeo e editada. A edição final do VE foi realizada com o uso do programa adobe première. Ressalta-se que durante todo o processo de desenvolvimento do vídeo a equipe de pesquisadores trabalhou sincronizada com a empresa contratada.

Para concepção dos personagens e cenários utilizou-se a técnica tradicional de desenho e colorido no Photoshop ${ }^{\circledR}$ utilizando a mesa digital Cintiq $^{\circledR} 12 \mathrm{WX}$, em seguida estes foram transformados em imagens tridimensionais, através do programa 3D Max $^{\circledR}$ e Z-Brush ${ }^{\circledR}$ (Figuras 1, 2 e 3). Os personagens virtuais desenvolvidos foram: o Francisco, 21 anos com paraplegia traumática completa e Luíza, 18 anos paraplegia incompleta. Os personagens, ambiente e objetos passaram pelo processo de renderizaçáo gráfica por meio do aplicativo $3 D$ Studio Max ${ }^{\circledR}$. Esse processo consiste em criar 
imagens e animaçôes tridimensionais em busca de atingir um grau maior de realismo. Para criação das ilustraçóes, coloraçáo dos objetos e o tratamento das imagens foi utilizado o programa adobe Photoshop ${ }^{\circledR}$. Para o motion design do vídeo, ou seja, a criação de imagens em movimento, foram utilizados os programas Adobe $e^{\circledR}$ flash e Adobe $e^{\circledR}$ after effects.

\section{Validação do roteiro}

Os dezoito juízes que participaram do processo de validação de conteúdo do roteiro procediam de diferentes centros brasileiros de referência em reabilitação vesical, além de universidades com estudos voltados para este tema e/ou produção de hipermídias e tecnologias educacionais. Entre os estados de procedência estavam: São Paulo, Santa Catarina, Rio de Janeiro, Minas Gerais, Bahia, Ceará, Maranhão, Mato Grosso do Sul e Distrito Federal.
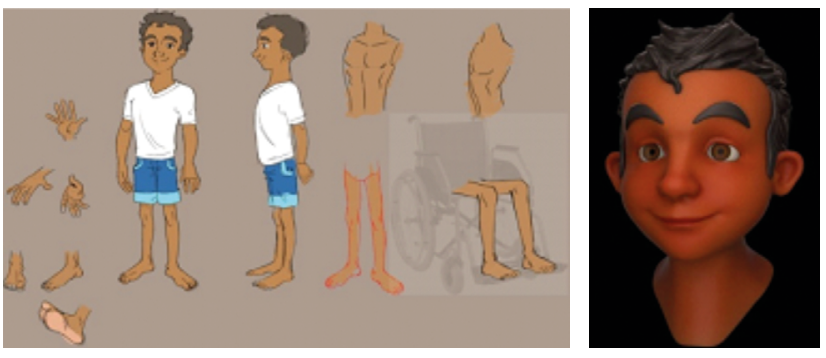

Figura l. Etapas de criação do personagem Francisco, versão modelagem e versão renderizada.
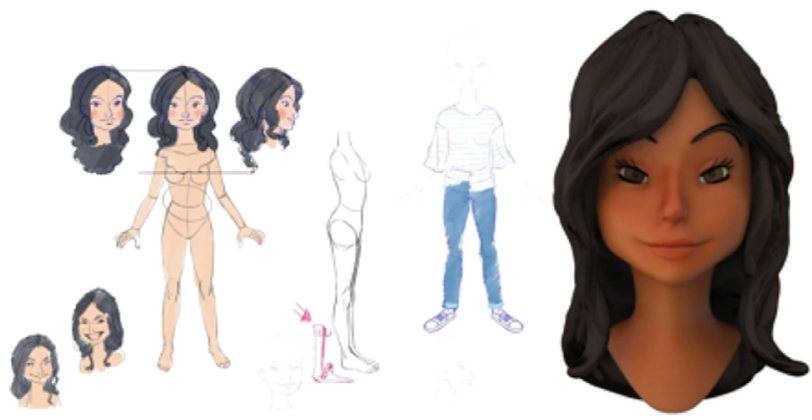

Figura 2. Etapas de criação da personagem Luísa, versão modelagem e versão renderizada.
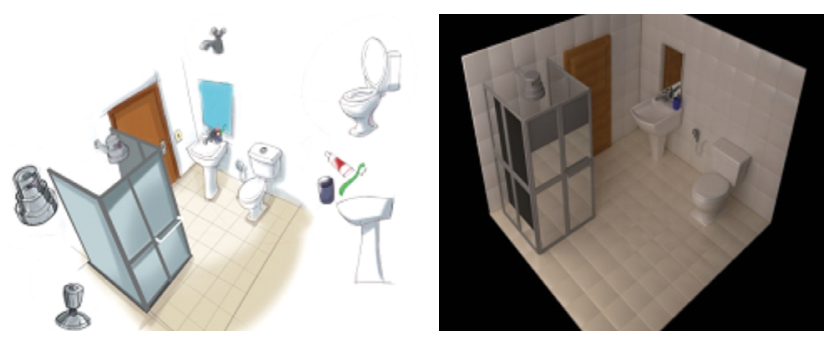

Figura 3. Etapas de criação do banheiro da casa de Francisco, versão modelagem e versão renderizada.
Em relação ao sexo dos juízes, observou-se predomínio de mulheres $17(94,4 \%)$ e a idade da amostra variou de 30 a 60 anos, com uma média de 38,7 anos $( \pm 8,3), 15$ participantes $(83,3 \%)$ eram enfermeiros.

O tempo de formaçáo variou entre cinco a 39 anos, com uma média de 15,2 anos de formação $( \pm 9,2)$. Atuavam na área de reabilitação de pessoas com deficiência 14 participantes $(77,8)$ e quatro participantes $(22,2 \%)$ trabalham na área de desenvolvimento de hipermídias e tecnologias educacionais.

Em relação à titulação acadêmica, 14 participantes $(77,8 \%)$ possuíam especialização, $13(72,2 \%)$ mestrado, oito $(44,4 \%)$ doutorado e dois $(11,1 \%)$ com pós-doutorado. Destes, nove $(50,0 \%)$ atuavam a mais de cinco anos na área de reabilitaçáo. Todos avaliadores afirmaram ter participado de algum evento científico nos últimos dois anos relacionado à sua área de atuação.

A seguir, na Tabela 1 , os dados seráo apresentados de acordo com cada seção do instrumento. Apesar do índice de concordância de todos os quesitos avaliados terem sido acima de $70,0 \%$, as sugestôes realizadas pelos juízes foram analisadas e a maioria foi acatada, principalmente aquelas relacionadas à necessidade aprimoramento da linguagem verbal.

\section{Validação do vídeo educativo}

Foram convidados os mesmos juízes que participaram da validação do roteiro para a validação do vídeo educativo, destes, 17 profissionais aceitaram participar dessa segunda fase da pesquisa.

Houve um número maior de mulheres 14 (82,3\%) e três participantes $(17,6 \%)$ homens. A variação da idade entre os participantes foi de 31 a 60 anos, com uma média de 39,1 anos $( \pm 7,5)$. A maioria dos participantes $(88,2 \%)$ eram enfermeiros, uma terapeuta ocupacional $(5,9 \%)$ e um médico $(5,9 \%)$.

Tabela 1. Distribuição das respostas dos juízes aos quesitos presentes na validação do roteiro do vídeo educativo.

\begin{tabular}{l|c|c|c} 
Quesitos & $\begin{array}{c}\text { Concordo/ } \\
\text { concordo } \\
\text { totalmente } \\
(\%)\end{array}$ & $\begin{array}{c}\text { Nem } \\
\text { concordo/ } \\
\text { nem } \\
\text { discordo (\%) }\end{array}$ & $\begin{array}{c}\text { Discordo/ } \\
\text { discordo } \\
\text { totalmente } \\
\text { (\%) }\end{array}$ \\
\hline Objetivos & 96,2 & - & 3,71 \\
\hline Conteúdo & 91,0 & 3,9 & 5,5 \\
\hline Relevância & 98,1 & - & 1,8 \\
\hline Ambiente & 75,0 & 19,4 & 5,5 \\
\hline $\begin{array}{l}\text { Linguagem } \\
\text { verbal }\end{array}$ & 71,1 & 17,7 & 11,1 \\
\hline $\begin{array}{l}\text { Inclusão } \\
\text { tópicos }\end{array}$ & 97,6 & 0,79 & 01,5 \\
\hline
\end{tabular}


O tempo de formaçáo teve variação de quatro a 40 anos, com média de 15,8 anos de formação $( \pm 8,7)$. Treze participantes $(76,5 \%)$ trabalhavam na área de reabilitação e quatro $(23,5 \%)$ trabalhavam na área de desenvolvimento de hipermídias e tecnologias educacionais.

Em relação à titulação acadêmica, 14 participantes $(82,3 \%)$ possuíam especialização, 10 (58,8\%) possuíam mestrado, oito $(47,1 \%)$ doutorado e um $(5,9 \%)$ com pós-doutorado. Destes, nove $(47,0 \%)$ trabalhavam há mais de cinco anos na área de reabilitação e todos os juízes afirmaram ter participado de algum evento científico nos últimos dois anos relacionados à sua área de atuação.

A validação do vídeo educativo (Tabela 2) atingiu um total de $97,4 \%$ de concordância entre os juízes nas questóes avaliadas $(84,09 \%$ de concordância no atributo "concordo totalmente", $13,3 \%$ no atributo "concordo"), sete respostas $(2,3 \%)$ como "nem concordo nem discordo" e apenas uma resposta $(0,3 \%)$ como "discordo". A questão "discordo totalmente" não foi assinalada.

Apesar da concordância dos juízes nos quesitos avaliados, as sugestóes para a modificação no roteiro visaram, na maioria, a adequação da linguagem utilizada para o público-alvo, a inclusão de informaçôes relacionadas à técnica do autocateterismo vesical intermitente feminino e a diferenciação entre o cateter hidrofílico e os cateteres plásticos. Houve também sugestôes quanto à reformulação conceituais sobre a anatomia do sistema urinário e a fisiopatologia da BN.

\section{DISCUSSÃO}

A elaboração de um VE para o ensino do autocateterismo vesical intermitente, voltado para pessoas com $\mathrm{BN}$, busca preencher uma lacuna em relação à escassez de vídeos educativos para o ensino dessa técnica no contexto brasileiro,

Tabela 2. Distribuição das respostas dos juízes de acordo com os quesitos do roteiro de validação do vídeo educativo.

\begin{tabular}{l|c|c|c} 
Quesitos & $\begin{array}{c}\text { Concordo/ } \\
\text { concordo } \\
\text { totalmente } \\
(\%)\end{array}$ & $\begin{array}{c}\text { Nem } \\
\text { concordo/ } \\
\text { nem } \\
\text { discordo (\%) }\end{array}$ & $\begin{array}{c}\text { Discordo/ } \\
\text { discordo } \\
\text { totalmente } \\
\text { (\%) }\end{array}$ \\
\hline Funcionalidade & 100,0 & - & - \\
\hline Usabilidade & 96,0 & 3,9 & - \\
\hline Eficiência & 97,0 & 2,9 & - \\
\hline Técnica & 100,0 & - & - \\
audiovisual & 94,1 & 5,8 & - \\
\hline Ambiente & 97,0 & 1,9 & 0,9 \\
\hline Procedimentos & & &
\end{tabular}

conforme verificado na avaliação dos vídeos disponíveis na internet no tema do estudo.

A versão final e validada do VE nesse estudo tem duração de 11 minutos e 19 segundos, cumprindo a recomendação de outros autores que sugerem que um vídeo não deverá ultrapassar 15 minutos, podendo tornar-se cansativo e induzir à dispersão da atenção do espectador ${ }^{(8,10,22)}$.

A validação do roteiro obteve a maioria $(91,1 \%)$ de respostas nos atributos "concordo totalmente" e "concordo", apresentando um resultado parecido com estudos de elaboração e validação de vídeos educativos virtuais ${ }^{(8,21)}$. Nesse sentido, assim como estudos anteriores, este estudo reforça a necessidade do processo de validação do roteiro e do VE, com a participação de experts, tendo como objetivo a melhoria dos conceitos abordados nas cenas, observaçóes para melhoria dos pontos de difícil compreensão ao público-alvo e sugestôes referentes à inclusão ou exclusão de informaçôes.

Apesar da concordância geral dos juízes nos quesitos avaliados, as sugestóes para a modificação no roteiro visando a adequação da linguagem foram acatadas. A adaptação da linguagem engloba a substituição de termos técnicos utilizados pelos profissionais de saúde e a compreensão dos aspectos culturais da linguagem de determinada população, tornando a comunicaçáo entre os pares efetiva ${ }^{(14,15)}$. Assim, a adequação da linguagem na produção do material educativo, necessária neste estudo assim como em anteriores, visa melhorar a compreensão do tema e é amplamente recomendada na produção de materiais para suporte da educação em saúde ${ }^{(15,23,24)}$.

Outra sugestão foi a inclusão de informaçóes referentes à mensuração do volume de urina para fazer o cálculo de ingesta. Essa sugestão foi acatada, com a justificativa de que a mensuração do volume urinário é necessária para avaliação e adaptação da frequência de realização do autocateterismo vesical intermitente às necessidades de cada pessoa ${ }^{(25)}$. Além disso, a mensuração de urina permite ao indivíduo avaliar os possíveis sinais de infecção através da avaliação da cor, cheiro, presença de resíduos e volume drenado ${ }^{(26)}$.

Em relação à inserção do cateter, alguns juízes apontaram para a necessidade de descrever o posicionamento dos homens durante o procedimento, para evitar o aparecimento de complicaçóes relacionadas ao processo. Embora o storyboard contemplasse informaçóes sobre o posicionamento do pênis para execução do procedimento técnico, o roteiro não contava com a narração que representasse essa etapa. Diante dessa demanda, foi acrescentado no vídeo uma figura que apresenta nitidamente o posicionamento do pênis em $90^{\circ}$ durante a inserção do cateter, visando prevenir lesôes uretrais ${ }^{(27)}$.

Algumas sugestóes sobre a sequência da higienização feminina, que deveria ser iniciada pelos grandes lábios, pequenos lábios e por último o meato uretral foram também apresentadas pelos experts. No entanto, referências atuais foram 
consultadas e não foi encontrada tal sequência invertida para a realização da técnica do autocateterismo vesical intermitente com a técnica limpa ${ }^{(28,29)}$. Deste modo, foi mantida a sequência iniciando a higienização feminina do meato uretral para os grandes lábios, considerando o princípio básico de iniciar a higiene sempre partindo da área menos contaminada para a mais contaminada, visando reduzir o número de microrganismos, e evitar trazê-los para o meato uretral ${ }^{(28,29)}$.

A avaliaçáo do item relacionado à usabilidade apontou questionamentos quanto à facilidade do uso do vídeo pela populaçáo e os avaliadores afirmaram que o uso da ferramenta educativa será benéfico apenas para as pessoas que tenham acesso ao computador e à internet. Por outro lado, é sabido que um número cada vez maior de pessoas possui acesso à internet no Brasil, segundo o Instituto Brasileiro de Geografia e Estatística (IBGE), sendo que há cobertura em 69,3\% dos domicílios brasileiros $^{(30)}$. Com isso, este VE poderá ter um alcance em todas as regióes brasileiras e não apenas na região sudeste onde foi desenvolvido e, acompanhando a crescente popularização dos dispositivos móveis e do acesso à internet, tem potencial de grande disseminação no meio online.

Atualmente, as ferramentas educativas virtuais visam transformar o aprendizado de apenas uma simples transferência de informaçóes e de habilidades para a construção do conhecimento pautado nas experiências, vivências e demandas pessoais. Apesar disso, o uso do VE deve ser validado por profissionais especialistas no tema a ser abordado, visto que esses materiais são ferramentas facilitadoras para a comunicação e orientação da população, possibilitando a padronização do ensino de determinada temática ${ }^{(31)}$.

Os juízes também sugeriram ressaltar a diferenciação do cateter hidrofílico e do cateter plástico, permitindo que a pessoa adquira conhecimentos para a escolha do dispositivo junto à equipe de saúde. Os cateteres hidrofílicos possuem propriedades que permitem lubrificação após contato com meio aquoso, e essa tecnologia faz com que tais cateteres deslizem mais facilmente pela uretra e previna traumas, embora estes dispositivos sejam mais $\operatorname{caros}^{(3,32)}$. Os cateteres de plástico demandam o uso de lubrificantes antes da inserção no meato uretral, além disso, alguns pacientes os reutilizam por um período de até de uma semana ${ }^{(12,32,33)}$. O lubrificante pode ser aplicado no cateter ou, no caso dos homens, pode ser aplicado diretamente na uretra ${ }^{(3,32)}$. Nesse aspecto, o uso de cateteres pré-lubrificados representa uma praticidade e economia de tempo durante a realização do ACVI. No Brasil, o uso de cateteres plásticos é mais comum, por serem disponibilizados nos serviços de saúde gratuitamente e devido às condiçóes socioeconômicas da população em geral ${ }^{(12)}$. No entanto, a apresentaçáo de diferentes opçóes de cateteres aos usuários permite que eles entendam as diferenças, os benefícios de cada um e possam optar na escolha do cateter que mais lhes convém. Deste modo, apesar de o VE já possuir este conteúdo, foi inserida mais uma figura mostrando a diferença entre os dois tipos de cateter.

O desenvolvimento deste estudo teve algumas limitaçôes, entre elas, a dependência do serviço de terceiros para o desenvolvimento da tecnologia 3D para a animaçáo e áudio do vídeo, que limitou a riqueza de detalhes devido ao desafio de troca de conhecimento e comunicação entre os profissionais envolvidos. Nesse último fator, uma sugestão seria contar com profissionais de tecnologia com conhecimento também na área de saúde, como os profissionais da informática biomédica. Apesar das limitaçóes do estudo, o vídeo produzido teve uma avaliação positiva durante o processo de validação e poderá contribuir para o cumprimento do objetivo para o qual o mesmo foi proposto.

\section{CONCLUSÃO}

A construção de um vídeo educativo é um processo longo e sistematizado o qual demanda esforços por parte dos pesquisadores na busca da melhor literatura científica sobre o tema, na seleção de expertises na temática e no rigor metodológico. Este estudo evidencia a necessidade da validação prévia do roteiro do VE e também do VE por juízes experts para se conseguir um produto com uma concordância satisfatória e que atenda às necessidades do público alvo. Além disso, o desenvolvimento de vídeos educativos com o uso de tecnologia 3D demanda apoio financeiro.

A expectativa com este VE, resultado deste estudo, é contribuir na capacitação de pessoas com $\mathrm{BN}$ para o ACVI, incluindo profissionais de saúde e estudantes de enfermagem, por meio da democratização do conhecimento na internet. A disponibilização desse VE em ambiente virtual pretende promover o acesso livre nas diferentes regióes do Brasil. Além de incentivar e embasar metodologicamente o desenvolvimento de outros vídeos educativos na área da saúde. A versão final do vídeo poderá ser visualizada brevemente no portal de informaçóes sobre saúde e inclusão para pessoas com deficiência: www.demaisinformacao.com.br e no site do e-aulas Portal de vídeo aulas da USP: http://eaulas.us.br/ portal/video.action?idltem $=8753$.

\section{REFERÊNCIAS}

1. Liao L. Evaluation and Management of Neurogenic Bladder: What Is New in China? Int J Mol Sci [Internet]. 2015 [acesso em: 25 jul. 2019];16(8):18580-600. Disponível em: https://doi.org/10.3390/ijms160818580.

2. Myers JB, Mayer EN, Lenherr S, (NBRG.org) NBRG. Management options for sphincteric deficiency in adults with neurogenic bladder. Transl Androl Urol [Internet]. 2016 [acesso em: 25 jul. 2019];5(1):145-57. Disponível em: https://doi.org/10.3978/j.issn.2223-4683.2015.12.11. 
3. Newman DK, Willson MM. Review of intermittent catheterization and current best practices. Urologic Nursing [Internet]. 2011 [acesso em: 25 jul. 2019];31(1):12-28, 48; quiz 29. Disponível em: https:// doi.org/10.7257/1053-816X.2012.31.1.12.

4. Hill TC, Baverstock R, Carlson KV, Estey EP, Gray GJ, Hill DC et al. Best practices for the treatment and prevention of urinary tract infection in the spinal cord injured population: The Alberta context. Can Urol Assoc J [Internet]. 2013 [acesso em: 25 jul. 2019];7(3-4):12230. Disponível em: https://doi.org/10.5489/cuaj.337.

5. Faleiros F, Käppler C, Costa JN, Favoretto N, Pontes F. Predictive Factors for Intermittent Self-catheterization in German and Brazilian Individuals With Spina Bifida and Neurogenic Bladder Dysfunction. J Wound Ostomy Continence Nurs [Internet]. 2016 [acesso em: 25 jul. 2019];43(6):636-40. Disponível em: https:// doi.org/10.1097/WON.0000000000000272.

6. Faleiros F, Cordeiro A, Favoretto N, Käppler C, Murray C, Tate D. Patients with Spina Bifida and their Caregivers Feelings about Intermittent Bladder Catheterization in Brazil and Germany: A Correlational Study. Rehabil Nurs [Internet]. 2015 [acesso em: 25 jul. 2019];42(4):175-79. Disponível em: https://doi. org/10.1002/rnj.223.

7. Souza VD Jr, Mendes IAC, Mazzo A, Santos CA, Andrade EMLR, Godoy S. Manual de telenfermagem para atendimento ao usuário de cateterismo urinário intermitente limpo. Esc Anna Nery [Internet]. 2017 [acesso em: 25 jul. 2019];21(4):e20170188. Disponível em: https://doi.org/10.1590/2177-9465ean-2017-0188.

8. Ferreira MVF, Godoy S, Góes F, Rossini FP, Andrade DA. Câmera e ação na execução do curativo do cateter venoso central. Rev Latino-Am Enfermagem [Internet]. 2015 [acesso em: 25 jul. 2019];23(6):1181-6. Disponível em: https://doi.org/10.1590/0104-1169.0711.2664.

9. Campoy LT, Rabeh SAN, Castro FFS, Nogueira PC, Terçariol CAS. Bowel rehabilitation of individuals with spinal cord injury: video production. Rev Bras Enferm [Internet]. 2018 [acesso em: 25 jul. 2019];71(5):2376-82. Disponível em: https://doi. org/10.1590/0034-7167-2017-0283.

10. Fleming SE, Reynolds J, Wallace B. Lights... camera... action! a guide for creating a DVD/video. Nurse Educ [Internet]. 2009 [acesso em: 25 jul. 2019];34(3):118-21. Disponível em: https://doi. org/10.1097/NNE.0b013e3181a0270e.

11. Faleiros F, Pelosi G, Warschausky S, Tate D, Käppler C, Thomas E. Factors Influencing the Use of Intermittent Bladder Catheterization by Individuals with Spina Bifida in Brazil and Germany. Rehabil Nurs [Internet].
2016 [acesso em: 25 jul. 2019];43(1):46-51. Disponível em: https://doi.org/10.1002/rnj.302.

12. Faleiros F, Toledo C, Gomide MFS, Faleiros R, Käppler C. Right to health care and materials required for intermittent catheterization: a comparison between Germany and Brazil. Quality in Primary Care [Internet]. 2015 [acesso em: 25 jul. 2019];23(3):127-33. Disponível em: http://primarycare.imedpub.com/rightto-health-care-and-materials-requiredfor-intermittentcatheterization-a-comparisonbetween-germany-andbrazil.pdf.

13. Fernandes ICF, Siqueira KM, MA B. Avaliação de vídeos sobre a técnica inalatória na asma infantil: educativos ou midiáticos? Rev Eletr Enf [Internet]. 2018 [acesso em: 25 jul. 2019];20(09):1-10. Disponível em: https:// doi.org/10.5216/ree.v20.48789.

14. Fonseca LM, Dias DM, Góes FSN, Seixas CA, Scochi CG, Martins JC et al. Development of the e-Baby serious game with regard to the evaluation of oxygenation in preterm babies: contributions of the emotional design. Comput Inform Nurs [Internet]. 2014 [acesso em: 25 jul. 2019];32(9):428-36. Disponível em: https://doi. org/10.1590/2177-9465-ean-2017-0424.

15. Favoretto NB, Faleiros F, Lopes F, Freitas G, Käppler C. Online health forum as a support for people who perform intermittent vesical catheterization. Texto \& Contexto - Enfermagem [Internet]. 2019 [acesso em: 11 jan. 2020];28:28:e20180263. Disponível em: https://dx.doi.org/10.1590/1980-265x-tce-2018-0263.

16. Barbosa RM, Bezerra AK. Validation of an educational video for the promotion of attachment between seropositive HIV mother and her child. Rev Bras Enferm [Internet]. 2011 [acesso em: 25 jul. 2019];64(2):328-34. Disponível em: https://doi. org/10.1590/S0034-71672011000200017.

17. Freitas LV, Teles LMR, Lima TM, Vieira NFC, Barbosa RCM, Pinheiro AKB, et al. Exame físico no pré-natal: construção e validação de hipermídia educativa para a Enfermagem. Acta Paul Enferm [Internet]. 2012 [acesso em: 25 jul. 2019];25(4):581-8. Disponível em: https://doi.org/10.1590/S0103-21002012000400016.

18. Fehring RJ. Methods to validate nursing diagnoses. Heart Lung [Internet]. 1987 [acesso em: 25 jul. 2019];16(6Pt1):625-9. Disponível em: https:// epublications.marquette.edu/cgi/viewcontent. cgi? article $=1026 \&$ context $=$ nursing $\mathrm{fac}$

19. Fernández-Donaire L, Romero-Sánchez JM, PalomaCastro O, Boixader-Estévez F, Porcel-Gálvez AM. The Nursing Diagnosis of "Death Anxiety": Content Validation by Experts. Int J Nurs Knowl [Internet]. 2018 [acesso em: 25 jul. 2019]; 30(4)211-18. Disponível em: https://doi.org/10.1111/2047-3095.12231. 
20. Bocchino A, Medialdea MJ, Lepiani I, Mejías C, Dueñas M. The Nursing Diagnosis Development of Unemployment Disorder: Content Validation With Nursing Experts. Int J Nurs Knowl [Internet]. 2017 [acesso em: 25 jul. 2019];28(4):184-91. Disponível em: https://doi.org/10.1111/2047-3095.12149.

21. Góes FSN, Fonseca LMM, Leite AM, Furtado MCC, Scochi CGS. Development and evaluation of an interactive virtual learning object on the diagnostic reasoning in nursing applied to preterm newborns. Procedia - Social and Behavioral Sciences [Internet]. 2012 [acesso em: 25 jul. 2019];46:617-23. Disponível em: https://doi.org/10.1016/j.sbspro.2012.05.173.

22. Carvalho EC, Stina APN, Marmol MT, Garbin LM, Braga FTMM, Moreli L, et al. Efeito de vídeo educativo no comportamento de higiene bucal de pacientes hematológicos. Rev Eletr Enf [Internet]. 2014 [acesso em: 25 jul. 2019];16(2):304-11. Disponível em: https://doi.org/10.5216/ree.v16i2.23300.

23. Nisbet G, Jorm C, Roberts C, Gordon CJ, Chen TF. Content validation of an interprofessional learning video peer assessment tool. BMC Med Educ [Internet]. 2017 [acesso em: 25 jul. 2019];17(258). Disponível em: https://doi.org/10.1186/s12909017-1099-5.

24. Brown T, Goldman SN, Persell SD, Lee JY, Doan CT, Stephens Q, et al. Development and evaluation of a patient education video promoting pneumococcal vaccination. Patient Educ Couns [Internet]. 2017 [acesso em: 25 jul. 2019];100(5):1024-7. Disponível em: https://doi.org/10.1016/j.pec.2016.12.025.

25. González MSJ, Fernández MP. Incontinencia y trastornos miccionales: ¿qué podemos hacer? Rev Pediatr Aten Primaria [Internet]. 2009 [acesso em: 25 jul. 2019];11(44):e1-e30. Disponível em: http://scielo. isciii.es/scielo.php?script $=$ sci_arttext $\&$ pid $=S 1139$ 76322009000500012\&lng=es.
26. Campos CVS, Silva KL. Cateterismo vesical intermitente realizado pelos cuidadores domiciliares em um serviço de atenção domiciliar. Revista Mineira de Enfermagem [Internet]. 2013 [acesso em: 25 jul. 2019];17(4):753-70. Disponível em: https://doi. org/10.5935/1415-2762.20130056.

27. Gonzalez Chiappe S, Lasserre A, Chartier Kastler E, Falchi A, Blaizeau F, Blanchon T, et al. Use of clean intermittent self-catheterization in France: A survey of patient and GP perspectives. Neurourol Urodyn [Internet]. 2016 [acesso em: 25 jul. 2019];35(4):528-34. Disponível em: https://doi.org/10.1002/nau.22752.

28. Perry AG, Potter PA. Guia completo de procedimentos e competências de enfermagem. Rio de Janeiro: Elsevier; 2012, 640p.

29. Potter PA, Perry AG. Fundamentos de enfermagem. $8^{\mathrm{a}}$ ed. Rio de Janeiro: Elsevier; 2013.

30. IBGE. Pesquisa Nacional por Amostra de Domicílios Contínua (PNAD) [Internet]. 2018. [acesso em: 25 jul. 2019]. Disponível em: https:// agenciadenoticias.ibge.gov.br/media/com mediaibge/ arquivos/49bcf11e47179d434bda979434770b0b.pdf.

31. Fonseca LMM, Leite AM, Mello DF, Silva MAI, Lima RAG, Scochi CGS. Tecnologia educacional em saúde: contribuiçôes para a enfermagem pediátrica e neonatal. Esc Anna Nery [Internet]. 2011 [acesso em: 25 jul. 2019];15(1):190-6. Disponível em: https://doi. org/10.1590/S1414-81452011000100027.

32. NHS NUH. Clinical Guidelines: Urinary Catheterisation Guidelines. Nottingham: Nottingham University Hospitals; 2011, 40p.

33. Krassioukov A, Cragg JJ, West C, Voss C, KrassioukovEnns D. The good, the bad and the ugly of catheterization practices among elite athletes with spinal cord injury: a global perspective. Spinal Cord [Internet]. 2015 [acesso em: 25 jul. 2019];53(1):78-82. Disponível em: https:// doi.org/10.1038/sc.2014.208. 